\title{
Peripherally Inserted Central Venous Catheters Provide Safe and Easy Central Venous Access in Patients with Head and Neck Cancer
}

\author{
Ryosuke Sato ${ }^{1}$ Takumi Kumai ${ }^{1}$ Ryusuke Hayashi ${ }^{1}$ HirokiKomatsuda ${ }^{1}$ Kan Kishibe ${ }^{1}$ Miki Takahara ${ }^{1}$ \\ Akihiro Katada ${ }^{1}$ Tatsuya Hayashi ${ }^{1}$ Yasuaki Harabuchi ${ }^{1}$ \\ ${ }^{1}$ Department of Otolaryngology-Head and Neck Surgery, Asahikawa \\ Medical University, Asahikawa, Japan \\ Int J Pract Otolaryngol 2021;4:e29-e33. \\ Address for correspondence Ryosuke Sato, MD, Department of \\ Otolaryngology-Head and Neck Surgery, Asahikawa Medical \\ University, 2-1-1-1, Midorigaoka-Higashi, Asahikawa 0788510, Japan \\ (e-mail: rsato@asahikawa-med.ac.jp).
}

\begin{abstract}
Keywords

- central venous catheters

- peripheral catheterization

- catheter-related infections

- head and neck cancer

Purpose The use of peripherally inserted central venous catheters (PICCs) has increased recently; several reports have revealed that they can be easily and safely used in patients with various diseases. However, there are few reports on the use of PICCs in patients with head and neck cancer. This study was aimed at evaluating the safety and feasibility of use of PICCs in patients with head and neck cancer.

Materials and Methods We retrospectively analyzed the date of 118 PICC insertions in 85 patients with head and neck cancer from January 2014 to December 2017. The PICCs have been placed under ultrasound guidance in all cases.

Results The PICC puncture success rate was $95.2 \%$. Catheter-related bloodstream infection occurred in four cases. The most common complication necessitating PICC removal was suspected catheter-related bloodstream infection ( 24 cases). All cases with confirmed and suspected catheter-related bloodstream infection improved with administration of antimicrobial agents. Phlebitis occurred in five cases, in all of whom the PICC placement had been made via an antecubital vein; the condition improved without treatment in all five cases. Deep vein thrombosis occurred in two cases, both of which improved with oral anticoagulant therapy.

Conclusion This study demonstrated that the complications associated with ultrasound-guided PICC insertion are manageable, and improve with conservative treatment in the majority of cases. Therefore, use of PICCs may be considered for easy and safe central venous access in patients with head and neck cancer, because the insertion success rate was acceptable.
\end{abstract}

\section{Introduction}

Peripherally inserted central venous catheters (PICCs) are used for central venous access and placed from a peripheral vein of the upper arm into the superior vena cava. In recent years, the use of PICCs has been gradually increasing because they are able to reduce the incidence of serious adverse events, such as infection and pneumothorax, and their insertion is considered easier than that of conventional central venous catheters (CVCs) through the subclavian vein. received

June 22, 2020

accepted

October 9, 2020
DOI https://doi.org/

$10.1055 / \mathrm{s}-0041-1728747$. ISSN 2569-1783.

\footnotetext{
(C) 2021. The Author(s).

This is an open access article published by Thieme under the terms of the Creative Commons Attribution-NonDerivative-NonCommercial-License, permitting copying and reproduction so long as the original work is given appropriate credit. Contents may not be used for commercial purposes, or adapted, remixed, transformed or built upon. (https://creativecommons.org/ licenses/by-nc-nd/4.0/) Georg Thieme Verlag KG, Rüdigerstraße 14, 70469 Stuttgart, Germany
} 
Several studies have reported that the use of PICCs is associated with a lower rate of catheter-related bloodstream infection (CRBSI) and reduced incidence of serious adverse events than conventional CVC in patients with hematological malignancies and patients in surgical care units. ${ }^{1-3}$ Catheterrelated adverse events are major reasons for the prolongation and interruption of treatment. Thus, safe and easy central venous access is essential in patients with head and neck cancer. As there are few reports on PICC use in patients with head and neck cancer, the aim of this study was to evaluate the safety and availability of PICC insertion in this patient population to determine whether it bears the same benefits as observed for patients with other diseases.

\section{Materials and Methods}

\section{Patients and Measures}

This was a retrospective analysis of 118 PICCs inserted in 85 patients with head and neck cancer treated in our department from January 2014 to December 2017. Data on demographic features, diagnosis, treatment, insertion site, insertion success rate, catheter days, adverse events, the incidence rate of CRBSI (the total number of onset of CRBSI/the total catheter days of catheterization $\times 1,000$ ), and reasons for PICC removal were analyzed. All PICCs were inserted for the purpose of the administration of chemotherapy. PICCs were also used for fluid replacement, intravenous nutrition, and antibiotic therapy, as necessary. All patients were treated with systemic chemotherapy or chemoradiotherapy mainly composed of cisplatin or radiotherapy and concomitant intra-arterial chemotherapy (RADPLAT) during the PICC insertion period. The study followed the principles of the Declaration of Helsinki. Informed written consent was obtained from all participants and the study design was approved by an ethics review board (Asahikawa Medical University: 20054).

\section{Methods}

PICCs were placed with ultrasound-guided puncture under X-ray radioscopy with maximum barrier precaution conditions. The catheter tips were placed in the superior vena cava. PICCs were placed by well-trained operators, including residents supervised by senior operators. PICCs were placed from the antecubital vein at the elbow in the beginning and flowing PICCs were placed from the basilic vein of the nondominant upper arm whenever possible. Single-lumen 4-Fr Groshong PICC (Bard, Billerica, Massachusetts, United States) was utilized from January 2014 to August 2017, and single-lumen 4-Fr power PICC (Bard) was utilized from August 2017 to December 2017. These PICCs have a rounded, closed-ended tip with a three-way valve designed to prevent blood reflux. PICCs were flushed with $0.9 \%$ saline solution every day.

CRBSI was defined according to the Infectious Disease Society of America's Clinical Practice Guidelines for the Diagnosis and Management of Intravascular Catheter-related Infections (2009). ${ }^{4}$ CRBSI was diagnosed when the same microorganisms were isolated from at least one set of blood culture bottles and the catheter tip or isolated from two blood culture bottles. Suspected CRBSI was defined as fever with no microorganisms isolated either from any blood culture bottle or from a catheter tip, defervesced by the withdrawal of PICC and appropriate antibiotic therapy, and no other instances of fever were observed. Other fever was defined as a fever unrelated to the PICCs placement, such as aspiration pneumonitis, tumor fever, and postoperative transient fever.

\section{Results}

\section{Patient Background}

The characteristics of the patients with head and neck cancer are shown in - Table 1. The 118 PICCs were placed in 85 patients with head and neck cancer at multiple sites (men/women: 71/14). The median age was 67 years (range: $28-78$ years). Eleven patients received chemotherapy alone, 54 patients received chemoradiotherapy, and 20 patients received RADPLAT.

\section{Insertion Success Rate}

A total of 124 punctures were attempted during this period, and insertion was successful in 118 cases (95.2\%). In six cases (4.8\%), PICC failed due to the difficulty of vascular puncture or insertion of the guide wire, and the central venous catheter was inserted from the femoral vein. The median duration of PICC placement was 16 days (range: 3-75 days). The total duration of catheterization was 2,831 days. Except

Table 1 Characteristics of the patients

\begin{tabular}{|c|c|}
\hline \multicolumn{2}{|l|}{ Sex } \\
\hline Male (median age) & $71(67)$ \\
\hline Female (median age) & $14(64)$ \\
\hline \multicolumn{2}{|l|}{ Diagnosis } \\
\hline Hypopharyngeal cancer & $25(29.6 \%)$ \\
\hline Oropharyngeal cancer & $22(26.2 \%)$ \\
\hline Oral cavity cancer & $8(9.5 \%)$ \\
\hline Sinonasal cancer & $7(8.3 \%)$ \\
\hline Laryngeal cancer & $7(8.3 \%)$ \\
\hline Nasopharyngeal cancer & $6(7.2 \%)$ \\
\hline Salivary gland cancer & $5(5.9 \%)$ \\
\hline Unknown primary cancer & $3(3.6 \%)$ \\
\hline Temporal bone cancer & $2(2.4 \%)$ \\
\hline \multicolumn{2}{|l|}{ Treatment } \\
\hline Chemotherapy & $11(12.9 \%)$ \\
\hline Chemoradiotherapy & $54(63.5 \%)$ \\
\hline RADPLAT & $20(23.6 \%)$ \\
\hline \multicolumn{2}{|l|}{ Puncture site } \\
\hline Basilic vein & $100(84.7 \%)$ \\
\hline Antecubital vein & $15(12.7 \%)$ \\
\hline Cephalic vein & $3(2.6 \%)$ \\
\hline
\end{tabular}

Abbreviation: RADPLAT, radiotherapy and concomitant intra-arterial chemotherapy. 
Table 2 Complications and reasons for PICC withdrawal ${ }^{\mathrm{a}}$

\begin{tabular}{|l|l|l|l|}
\hline & $\begin{array}{l}\text { PICC } \\
\text { removal } \\
\text { (A) }\end{array}$ & $\begin{array}{l}\text { Non-PICC } \\
\text { removal } \\
\text { (B) }\end{array}$ & $(\mathrm{A})+(\mathrm{B})$ \\
\hline CRBSI & 3 & 1 & 4 \\
\hline $\begin{array}{l}\text { Suspicion } \\
\text { of CRBSI }\end{array}$ & 24 & 0 & 24 \\
\hline Other fever & 6 & 14 & 20 \\
\hline Phlebitis & 3 & 2 & 5 \\
\hline Leakage & 1 & 0 & 1 \\
\hline $\begin{array}{l}\text { Mild bleeding } \\
\text { at the } \\
\text { insertion site }\end{array}$ & 0 & 6 & 6 \\
\hline $\begin{array}{l}\text { Deep vein } \\
\text { thrombosis }\end{array}$ & 2 & 0 & 2 \\
\hline Total number & 39 & 23 & 62 \\
\hline
\end{tabular}

Abbreviations: CRBSI, catheter-related bloodstream infection; PICC, peripherally inserted central venous catheter.

${ }^{\mathrm{a} A}$ total of 62 adverse events (57 out of 118 PICCs) occurred.

for one PICC whose insertion site was unknown, 86 PICCs were inserted in the left upper arm (73.5\%) and 31 PICCs in the right upper arm (26.5\%). One hundred and three of the 118 PICCs were placed from a vein above the elbow (100 PICCs from the basilic vein, 3 PICCs from the cephalic vein). Fifteen of 118 PICCs were placed from the antecubital vein at the elbow. PICCs were inserted from the contralateral side of the upper arm in 9 of 118 cases (7.3\%) because of insertion difficulty. There were no adverse events during insertion.

\section{Late Adverse Events}

The adverse events and reasons for withdrawal of PICCs are shown in - Table 2. Fifty-seven out of 118 PICCs suffered from adverse events, including non-PICC removal (48.3\%). A total of 62 adverse events occurred during the period. Seventy-nine of 118 PICCs were removed because of the end of therapy (66.9\%), and 39 PICCs were removed because of adverse events (33.1\%), with the most common being suspicion of CRBSI (24 cases). True CRBSI occurred in four cases (CRBSI rate: 1.4 per 1,000 catheter days). These were due to the following microorganisms: Staphylococcus epidermidis (two cases), methicillin-susceptible Staphylococcus aureus (one case), and methicillin-resistant S. aureus (one case). All cases of CRBSI and of suspicion of CRBSI improved by administration of antimicrobial agents. Phlebitis occurred in five cases and the PICCs were removed in three of these. Phlebitis improved without treatment in all cases. PICCs were inserted from the antecubital vein at the elbow in all cases with phlebitis. Deep vein thrombosis at the upper arm occurred in two cases placed from the basilic vein, and the PICCs were removed in both cases. Deep vein thrombosis improved through oral anticoagulant therapy. Mild bleeding at the puncture site after PICC placement occurred in six cases but did not require additional treatment.

\section{Discussion}

In this study, we examined the safety and feasibility of PICC in patients with head and neck cancer and obtained promising results. Patients with head and neck cancer often require CVC placement for administration of high-dose chemotherapy, and CVCs are also useful for intravenous hyper alimentation and fluid replacement when the patients have severe mucositis due to radiotherapy and chemotherapy. CVCs are generally placed through the internal jugular vein because of ease of insertion and lower risk of mechanical adverse events. ${ }^{5}$ Because the internal jugular vein can be the tumor/treatment site in head and neck cancer, CVCs are conventionally placed through the subclavian vein or the femoral vein, which may cause serious adverse events. Catheterization through the subclavian vein was associated with the potential of pneumothorax, ${ }^{6}$ while catheterization through the femoral vein was associated with high risk of infection compared with other approaches. ${ }^{7}$

PICCs are conventionally placed from the elbow without ultrasonographic guidance. ${ }^{8}$ In conventional PICC puncture methods, the puncture vein is determined with palpation and external visual inspection. However, in some patients with obesity or poor vein condition, the success rate of PICCs placement was low. ${ }^{9}$ Repeated punctures may cause phlebitis and damage the local tissues. Ultrasound-guided PICC puncture can be used to directly observe and access the vein. 8,10 McMahon reported that PICC puncture using ultrasonography improved the success rate of PICC placement from 65 to $91 \%{ }^{11}$ Other reports have also described the overall success rate of PICC insertion at 94 and 95\% with ultrasound-guided puncture. $^{3,12}$ In the present study, the PICC insertion success rate was $95.2 \%$, and adverse events did not occur during insertion. Six cases failed due to the difficulty of vascular puncture or inserting the guide wires. These cases tended to be characterized by a reduction of the vascular diameter or vascular meandering by ultrasonography, although no detailed measurements were performed. Therefore, pre-scan ultrasonography had the possibility of being useful in the prediction of insertion success. However, further examination is necessary as for prediction of the insertion success.

Sixty-two cases of late adverse events occurred in 118 PICC placements, including 28 cases of CRBSI or suspicion of CRBSI. Because all patients with PICCs insertion were administered chemotherapy, mainly composed of high-dose cisplatin or superselective intra-arterial cisplatin infusion, they were considered to be immune-compromised. Nevertheless, all CRBSI cases improved with the administration of antimicrobial agents. Several reports indicated that the use of PICCs was associated with a lower CRBSI rate than the use of conventional CVCs because the surface of the upper arm is less colonized and less moist than other areas. ${ }^{1-3,13}$ Gunst et al reported that the incidence rate of CRBSI as observed in bacterial culture was 2.1 per 1,000 catheter days in PICCs and 6.0 per 1,000 catheter days in conventional CVCs in surgical care units. ${ }^{2}$ Sakai et al also reported that the incidence rate of CRBSI was 1.2 per 1,000 catheter days in PICCs and 5.3 per 1,000 catheter days in conventional CVCs in patients with hematological 
malignancies. ${ }^{1}$ In our cases, the incidence of CRBSI was acceptable (CRBSI rate: 1.4 per 1,000 catheter days). Only 4 patients were diagnosed with CRBSI by bacterial culture, and the 24 cases of suspicion of CRBSI could have been of noninfectious fever. When patients given PICCs have a fever, PICC removal is not necessarily required. ${ }^{4}$ In one patient with CRBSI in which the PICC was not removed, their infection was improved only by the administration of an antimicrobial agent. However, to prevent the possible exacerbation of the infection and interruption of the treatment by infection, we systematically removed the PICCs in patients with fever even if a diagnosis of CRBSI was not obtained. In this study, all cases with fever had a good course of infection by intervening at an early stage.

PICC placement is associated with deep vein thrombosis of the upper limb, which requires emergency treatment, and pulmonary embolism. A meta-analysis revealed a higher incidence rate of deep vein thrombosis with PICCs placement than with conventional CVCs placement (odds ratio: 2.55). ${ }^{14}$ However, this report showed no incidence of serious pulmonary embolism due to PICC placement. In terms of PICCs placement, the vascular diameter to puncture is small regardless of a vascular site. This is considered to cause deep vein thrombosis. Clinical data suggested that the large diameter of the catheter increased deep vein thrombosis rates because the presence of the catheter decreased blood flow to the vein. ${ }^{15}$ Therefore, we used small-caliber singlelumen PICCs to prevent deep vein thrombosis. An ultrasound-guided insertion may also reduce the incidence of PICC-related deep vein thrombosis due to reduced blood vessel damage at the time of puncture. ${ }^{16}$ In the present study, deep vein thrombosis occurred in only two cases placed from the basilica vein. Both patients were successfully treated with only the administration of an oral anticoagulant because the embolus was small. Deep vein thrombosis was suspected by clinical manifestations, such as local swelling or pain, and diagnosed by contrast-enhanced computed tomography (CT). Both patients with deep vein thrombosis could be diagnosed early by contrast-enhanced CT.

Furthermore, ultrasound-guided insertion from the upper limb reduces the incidence of phlebitis. ${ }^{15}$ PICCs are generally placed from a vein at the elbow, such as the antecubital vein without ultrasonographic guidance. Because the elbow joint movement increases the tension and friction of the duct, PICCs in the elbow increase the incidence of mechanical phlebitis. ${ }^{16,17}$ Therefore, PICCs should be placed from the basilic vein above the elbow with ultrasound-guided puncture to reduce the risk of deep vein thrombosis and phlebitis. In the present cases, all incidences of phlebitis occurred in PICCs placed from the antecubital vein (5/15 cases). Two out of five cases were improved without PICCs withdrawal. Phlebitis was characterized by local swelling, erythema, and tenderness along the tract of the catheter. Phlebitis is generally improved with a conservative treatment; however, in cases without improvements in findings, the PICC should be removed. ${ }^{18}$

Because we could not directly compare the use of PICCs with the use of conventional CVCs, it is not known whether adverse events of PICCs are lower than adverse events of conventional CVCs. However, no serious adverse events occurred and the incidence of CRBSI was low, despite the fact that all patients were treated with chemotherapy. Thus, PICC may be considered a safe and effective central venous access technique in this patient population. Prospective study that compares PICC and conventional CVC will be required to validate these findings.

\section{Conclusion}

The present study demonstrated that there was no evidence of serious adverse events caused by PICC placement. A high puncture success rate (95.2\%) was achieved with ultrasoundguided PICC puncture with a low incidence rate of deep vein thrombosis and phlebitis. The PICC puncture site did not interfere with the tumor/treatment site in patients with head and neck cancer. Thus, PICC is considered a safe and effective central venous access technique in this patient population.

\section{Funding}

This research did not receive any specific grant from funding agencies in the public, commercial, or not-forprofit sectors.

\section{Conflict of Interest}

None declared.

\section{Acknowledgment}

We would like to thank Editage (www.editage.com) for English language editing.

\section{References}

1 Sakai T, Kohda K, Konuma Y, et al. A role for peripherally inserted central venous catheters in the prevention of catheter-related blood stream infections in patients with hematological malignancies. Int J Hematol 2014;100(06):592-598

2 Gunst M, Matsushima K, Vanek S, Gunst R, Shafi S, Frankel H. Peripherally inserted central catheters may lower the incidence of catheter-related blood stream infections in patients in surgical intensive care units. Surg Infect (Larchmt) 2011;12(04):279-282

3 Bellesi S, Chiusolo P, De Pascale G, et al. Peripherally inserted central catheters (PICCs) in the management of oncohematological patients submitted to autologous stem cell transplantation. Support Care Cancer 2013;21(02):531-535

4 Mermel LA, Allon M, Bouza E, et al. Clinical practice guidelines for the diagnosis and management of intravascular catheter-related infection: 2009 update by the Infectious Diseases Society of America. Clin Infect Dis 2009;49(01):1-45

5 Frykholm P, Pikwer A, Hammarskjöld F, et al; Swedish Society of Anaesthesiology and Intensive Care Medicine. Clinical guidelines on central venous catheterisation. Acta Anaesthesiol Scand 2014; 58(05):508-524

6 Parienti JJ, Mongardon N, Mégarbane B, et al; 3SITES Study Group. Intravascular complications of central venous catheterization by insertion site. N Engl J Med 2015;373(13):1220-1229

7 O'Grady NP, Alexander M, Burns LA, et al; Healthcare Infection Control Practices Advisory Committee (HICPAC) (Appendix 1) Summary of recommendations: guidelines for the prevention of intravascular catheter-related infections. Clin Infect Dis 2011;52 (09):1087-1099

8 Wang Q Wang N, Sun Y. Clinical effect of peripherally inserted central catheters based on modified Seldinger technique under guidance of vascular ultrasound. Pak J Med Sci 2016;32(05):1179-1183 
9 Elen Hughes MM. PICC-related thrombosis: pathophysiology, incidence, morbidity and the effect of ultrasound-guided placement technique on occurrence in cancer patients. JAVA 2011;16:8-18

10 Gong P, Huang XE, Chen CY, Liu JH, Meng AF, Feng JF. Comparison of complications of peripherally inserted central catheters with ultrasound guidance or conventional methods in cancer patients. Asian Pac J Cancer Prev 2012;13(05):1873-1875

11 McMahon DD. Evaluating new technology to improve patient outcomes: a quality improvement approach. J Infus Nurs 2002;25 (04):250-255

12 Harter C, Ostendorf T, Bach A, Egerer G, Goldschmidt H, Ho AD. Peripherally inserted central venous catheters for autologous blood progenitor cell transplantation in patients with haematological malignancies. Support Care Cancer 2003;11(12):790-794

13 Roth RR, James WD. Microbial ecology of the skin. Annu Rev Microbiol 1988;42:441-464
14 Chopra V, Anand S, Hickner A, et al. Risk of venous thromboembolism associated with peripherally inserted central catheters: a systematic review and meta-analysis. Lancet 2013;382 (9889):311-325

15 Nifong TP, McDevitt TJ. The effect of catheter to vein ratio on blood flow rates in a simulated model of peripherally inserted central venous catheters. Chest 2011;140(01):48-53

16 Hashimoto Y, Fukuta T, Maruyama J, Omura H, Tanaka T. Experience of peripherally inserted central venous catheter in patients with hematologic diseases. Intern Med 2017;56(04):389-393

17 Allen AW, Megargell JL, Brown DB, et al. Venous thrombosis associated with the placement of peripherally inserted central catheters. J Vasc Interv Radiol 2000;11(10):1309-1314

18 Merrell SW, Peatross BG, Grossman MD, Sullivan JJ, Harker WG. Peripherally inserted central venous catheters: low-risk alternatives for ongoing venous access. West J Med 1994;160(01):25-30 